\title{
Design of the key Structure of Convolutional Neural Network Reconfigurable Accelerator Based on ASIC
}

\author{
Hongli Pan ${ }^{1, \text { a) }}$, Mingjiang Wang ${ }^{2, \text { b) }}$ and Jingqun $\mathrm{Li}^{2, \mathrm{c})}$ \\ School of Electronic and Information Engineering, Harbin Institute of Technology, Shenzhen 518000, China. \\ a) panhonglihit@163.com, ${ }^{\text {b) }}$ mjwang@hit.edu.cn, ${ }^{\text {c) }}$ 791500217@qq.com
}

\begin{abstract}
In this paper, we propose a reconfigurable architecture that supports various convolutional neural networks (CNNs) such as GoogLeNet and AlexNet. The proposed architecture mainly includes 24 parallel PEs (processing engines) for image data convolution processing, each engine containing 9x4 MAC (multiplier-accumulator) units. Through the combination of PE, this structure can support a variety of bit-width convolution operations, namely 8 bit $\times 8$ bit, 16 bit $\times 8$ bit, $16 \mathrm{bit} \times 16 \mathrm{bit}$. At the same time, it also supports a variety of sizes of convolution operation, that is, $1 \times 1,3 \times 3,5 \times 5,7 \times 7$. The architecture is synthesized using 65-nm TSMC technology and achieves a peak of 1105.9 GOPS at $640 \mathrm{MHz}, 1 \mathrm{~V}$, and a power consumption of $193 \mathrm{~mW}$. Compared with the existing AlexNet architecture, the proposed architecture improves the computational efficiency by $20 \%$ to $27.4 \%$.
\end{abstract}

Key words: Convolutional Neural Network, CNN, accelerator, GoogleNet, AlexNet.

\section{INTRODUCTION}

Deep learning shows excellent performance in various applications such as computer vision and voice processing. Convolutional neural networks can achieve unprecedented accuracy, such as object recognition, detection, and so on $[1,2]$. However, CNN-based applications face two important issues: computational complexity is much higher than traditional methods; parametric data transmission requires high storage bandwidth.

Improving CNN performance requires overcoming a number of computation-related issues. The traditional way to accelerate CNN is to use a GPU capable of high-speed matrix multiplication and various types of FPGAs (Field Programmable Gate Arrays). Some scholars point out that one way to continue to improve processor performance and energy cost is to develop domain-specific methods [3,4]. processor. Some researchers have proposed some ASIC-based (application specific integrated circuits) CNN accelerators. Many companies are developing artificial intelligence dedicated hardware that focuses on performance [5,6]. Develop an architecture that supports parallel processing of multiple PE units and increase the efficiency of CNN operations. It is of great significance to the practical application of deep learning.

This paper presents a multi-mode CNN accelerator that includes 24 reconfigurable processing engines (PEs). Each PE supports $9 \times 4$ MAC (multiply-accumulate) operations in parallel. Through the combination of PE, it supports different bit-width data and weights. Also supports different size convolution operations. Because convolution accounts for more than $90 \%$ of $\mathrm{CNN}$ operations, the article focuses on convolutional layer design. Compared with the existing research results, part of the performance is improved.

\section{DESIGN OF PE ARRAY STRUCTURE}

As shown in Figure 1, the structure of the PE array consists of $243 \times 3$ PE units. The PE unit supports different numbers of MAC operations in different modes. In the 8-bit by 8-bit mode, each PE unit supports $9 \times 4$ MAC operations; in the 16-bit by 8 -bit mode, $9 \times 2 \mathrm{MAC}$ are supported; in the 16-bit by 16-bit mode, 9 MAC are supported. 
The PE array architecture supports concurrent convolution operations for $1 \times 1,3 \times 3,5 \times 5$, and $7 \times 7$ convolutional templates.

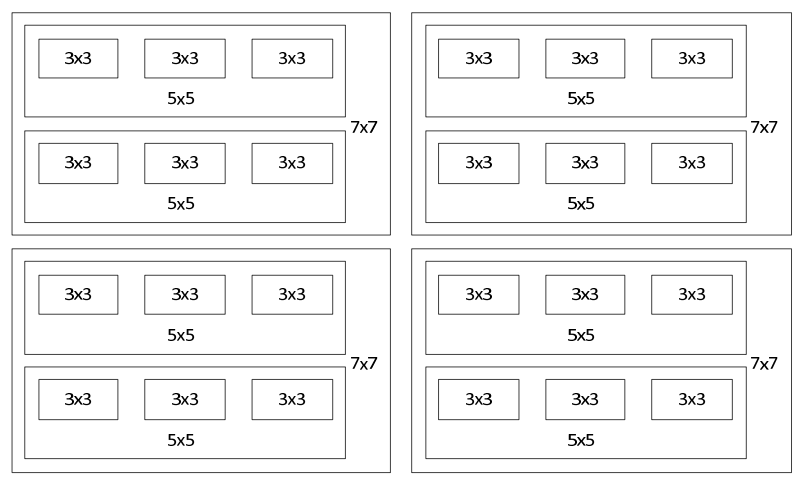

FIG. 1. Structure of PE Array

\section{Single Multi-Mode PE Structure.}

As shown in Figure 2, a PE contains 36 (4 x 9) Booth coding units, and 16 Wallace tree XXs (4 Wallace trees 00 03, 4 Wallace trees 10 13, 4 Wallace trees 20 23, and 4 Wallace trees 30 33.) Units, 4 Wallace trees_0 units, 2 Wallace trees_1 units, 1 Wallace tree_2 unit and 4 CPA_0 units, 2 CPA_1 units, and 1 CPA_2 unit. The data that can be processed is divided into 3 modes, 8 bit $\times 8$ bit, 16 bit $\times 8$ bit and $16 \mathrm{bit} \times 16$ bit. The 368 -bit image/feature data is input as a multiplicand to the Booth encoding unit, and the 36-parameter data is input as a multiplier. Each Booth encoding unit outputs 4 data d0, d4. Each of the 9 Booth encoding units is divided into a group, and all d0 of the 9 Booth encoding units in the group are connected to the input of Wallace tree_0; 9 Booth encoded d1 of the group are input to Wallace treeX1, and so on. Therefore, all Wallace treeXX inputs are bit-aligned, effectively reducing the number of columns in the Wallace treeXX array, thus saving circuit area. The output of each Wallace treeX0 X3 is used as the Wallace tree_0 input. The output of Wallace tree_0 acts as the input to CPA0 (Carry Spread Adder). The result of 4 sets of CPA0 is output as an 8 bit $\times 8$-bit result. The output of Wallace tree 0 is also used as the input of Wallace tree_1. The output of Wallace tree_1 is used as the input of CPA_1, and the output of CPA_1_0 and CPA_1_1 is output as the result of $16 \mathrm{bit} \times 8 \mathrm{bit}$. The output of Wallace tree_1 is also used as the input of Wallace tree $\overline{2}$. The output of Wallace tree_2 is output as the result of 16 bit $\times 16$ bit after the result obtained by CPA_2. The output of CPA_0,CPA_1, CPA_2 only takes the truncated 16-bit data, discards the remaining digits, and holds the $3 \times 3$ convolution result as 16 bits.

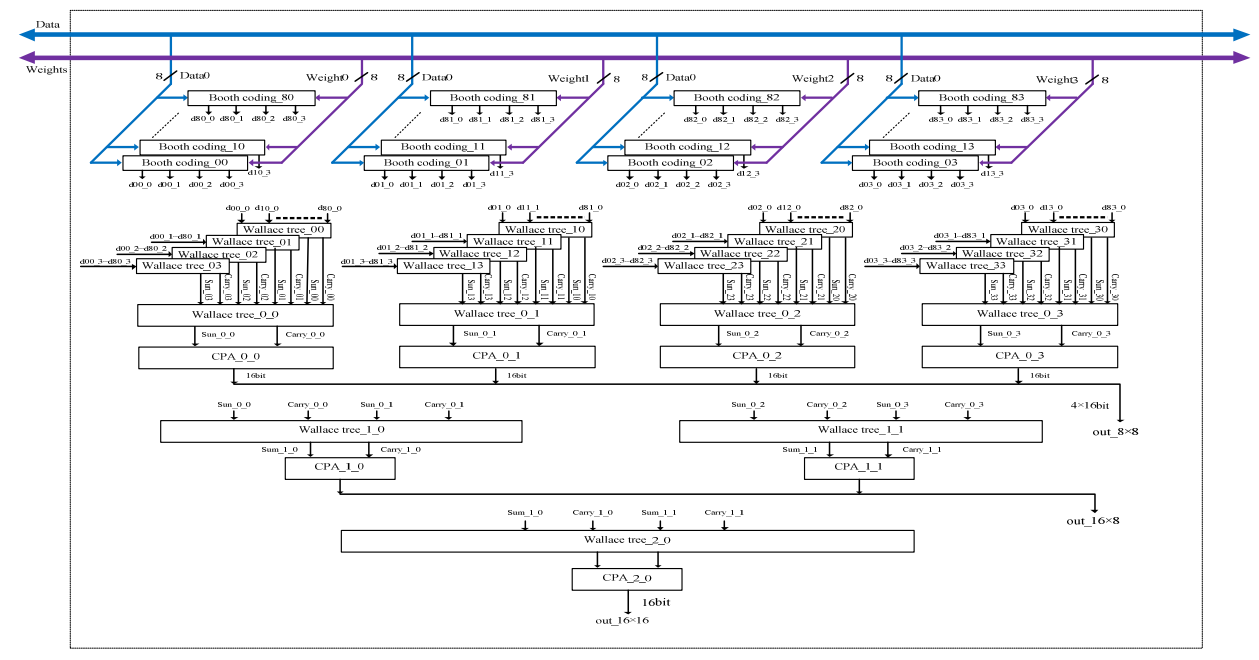

FIG. 2. Single multi-mode PE Structure 


\section{Combination of PE Arrays.}

As shown in Figure 3, three PEs are occupied for a $5 \times 5$ convolution. The output of each PE is the output of the four Wallace tree_0s in Figure 3, a total of eight. The outputs of carry0_0 and sum0_0 of the three PEs are collectively used as the input of Wallace treeA_0, three carry $0 \_1$ and three sum0_1 of three PEs are input as Wallace treeA_1, and so on. The outputs of the 8 Wallace treeA are taken as the input of 4 CPA_A, and the outputs of the 4 CPA_ $\bar{A}$ are $5 \times 5$ convolution results in the 8 bits $\times 8$-bit mode. The outputs of the 8 Wallace treeA are divided into 2 groups, which are respectively the input of Wallace treeB. The output of Wallace treeB is passed to two CPA_Bs, and the two outputs of CPA_B are the $5 \times 5$ convolution results in $16 \mathrm{bit} \times 8 \mathrm{bit}$ mode. The output of the two Wallace treeBs is also used as the input of the Wallace treeC. After that, the CPA_C results in a $5 \times 5$ convolution result in the 16 bits $\times 16$-bit mode. Figure 4 shows a $7 \times 7$ convolution with 6 PEs. It is composed of two $5 \times 5$ convolutions, and the structure is similar to a $5 \times 5$ convolution. It uses the 3 types of Wallace trees and 3 types of CPA to output $7 \times 7$ convolution results in 8 bit $\times 8$ bit, 16 bit $\times 8$ bit and 16 bit $\times 16$ bit modes, respectively.

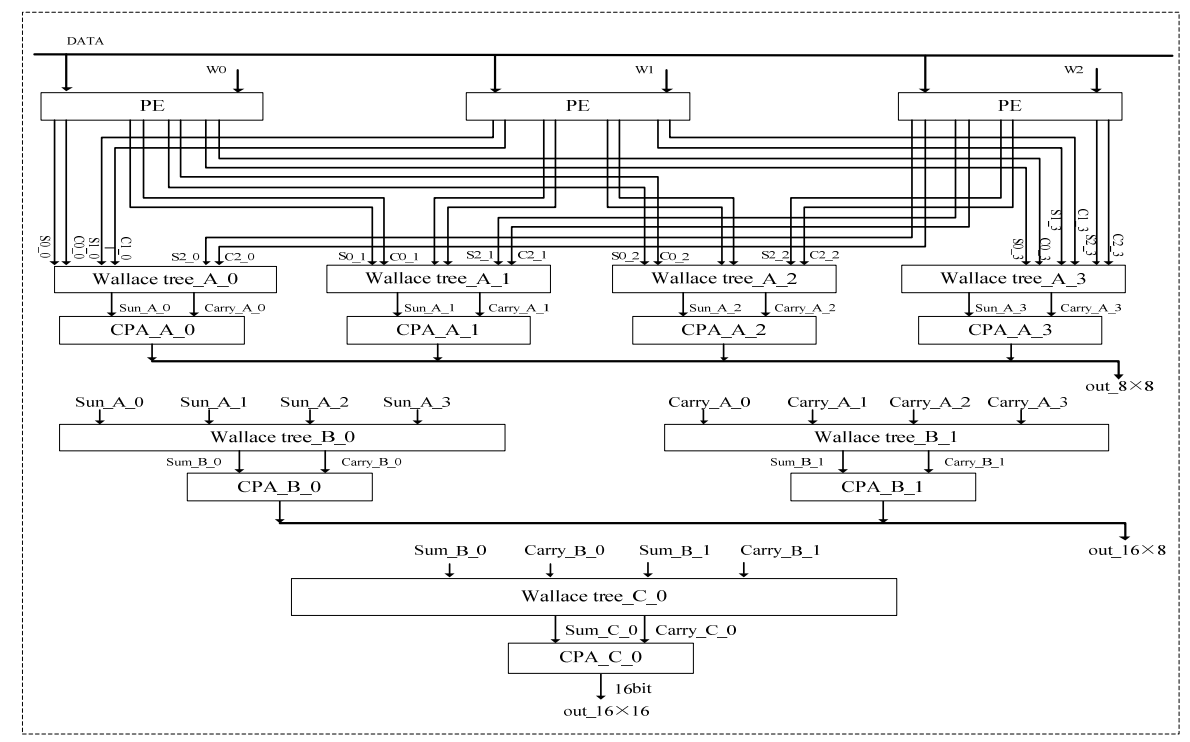

FIG.3. Combination of $5 \times 5$

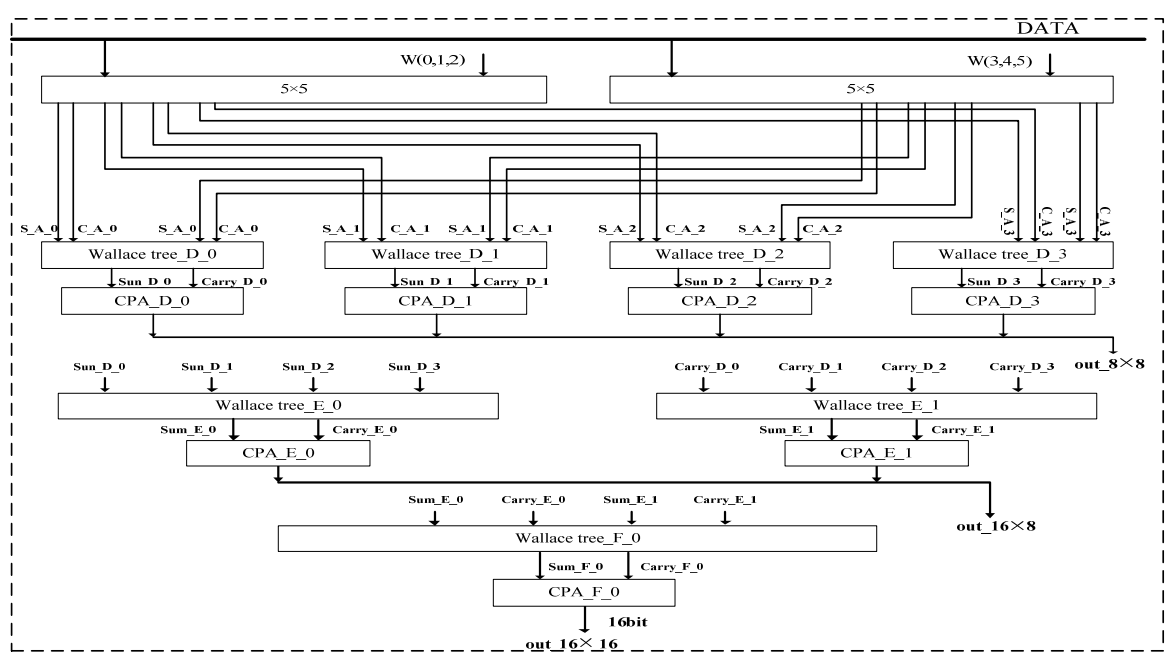

FIG. 4. Combination of $7 \times 7$ 


\section{EXPERIMENTAL RESULTS}

Using the 65nm TSMC CMOS technology, we synthesized the PE architecture of our design and achieved a core clock frequency of $640 \mathrm{MHz}$. PE array area of $1.23 \mathrm{~mm} 2$ and power consumption of $193.3 \mathrm{~mW}$. The maximum performance reached 1105.9 GOPS, the average performance reached 981.6 GOPS, and the computational efficiency reached $88.8 \%$. As shown in Table 1. Compared with the existing AlexNet architecture, this architecture improves computational efficiency by $20 \%$ to $27.4 \%$.

TABLE 1. Three Scheme comparing

\begin{tabular}{cccc}
\hline & Chen'16 [5] & Moons'17 [6] & Our design \\
\hline Technology & $65 \mathrm{~nm} \mathrm{LP}$ & $40 \mathrm{~nm} \mathrm{LP}$ & $65 \mathrm{~nm}$ \\
MAC per PE & 1 & 1 & $9 \times 4$ \\
Supply Voltage & $1 \mathrm{~V}$ & $0.85-0.92 \mathrm{~V}$ & $1 \mathrm{~V}$ \\
Nominal Frequency & $200 \mathrm{MHz}$ & $204 \mathrm{MHz}$ & $640 \mathrm{MHz}$ \\
Peak Performance & $67.2 \mathrm{GOPS}$ & $102 \mathrm{GOPS}$ & $1105.9 \mathrm{GOPS}$ \\
Average Performance (AlexNet) & $46.2 \mathrm{GOPS}$ & $62.6 \mathrm{GOPS}$ & $981.6 \mathrm{GOPS}$ \\
Computational Efficiency & $68.8 \%$ & $61.4 \%$ & $88.8 \%$ \\
\hline
\end{tabular}

\section{SUMMARY}

This paper presents a multi-mode high-performance CNN accelerator architecture that supports various convolutional neural networks such as GoogLeNet and AlexNet. This architecture supports $1 \times 1,3 \times 3,5 \times 5,7 \times 7$ convolutions in three-bit width $(8 \mathrm{bit} \times 8 \mathrm{bit}, 16 \mathrm{bit} \times 8 \mathrm{bit}, 16 \mathrm{bit} \times 16 \mathrm{bit})$ modes. The architecture includes $24 \mathrm{PEs}$, each with $9 \times 4$ MAC units. The design is synthesized in a $65-\mathrm{nm}$ TSMC and operates with a $1 \mathrm{~V} 640 \mathrm{MHz}$ core clock, consumes $193.3 \mathrm{~mW}$, and has an area of $1.23 \mathrm{~mm} 2$. Compared with ASICs based on AlexNet's existing similar architecture, the computational efficiency has increased by $20 \%$ to $27.4 \%$.

\section{REFERENCES}

1. Krizhevsky, A.; Sutskever, I.; Hinton, G. E. ImageNet Classification with Deep Convolutional Neural Networks. In Advances in Neural Information Processing Systems 25 (NIPS 2012); Advances in Neural Information Processing Systems; Lake Tahoe, Nevada, USA, December 3 - 8, 2012; Curran Associates, Inc.: 2012; Vol. 2, pp. 1097-1105.

2. He, K.; Zhang, X.; Ren, S.; Sun, J. Deep residual learning for image recognition. In 2016 IEEE Conference on Computer Vision and Pattern Recognition (CVPR); Proceedings of the IEEE Computer Society Conference on Computer Vision and Pattern Recognition; Las Vegas, NV, USA, 27-30 June 2016; IEEE: 2016; pp. 770-778.

3. Lee, Y.; Kim, H.; Park, E.; Yim, B.; Kim, H. Optimization for object detector using deep residual network on embedded board. In 2016 IEEE International Conference on Consumer Electronics-Asia (ICCE-Asia); Seoul, South Korea, 26-28 Oct. 2016; IEEE: 2016; pp. 1-4.

4. Rhu, M.; Gimelshein, N.; Clemons, J.; Zulfiqar, A.; Keckler, S. W. vDNN: Virtualized deep neural networks for scalable, memory-efficient neural network design. In 2016 49th Annual IEEE/ACM International Symposium on Microarchitecture (MICRO); Taipei, Taiwan, 15-19 Oct. 2016; IEEE: 2016; pp. 1-13.

5. Chen, Y.-H.; Krishna, T.; Emer, J.; Sze, V. Eyeriss: An energy-efficient reconfigurable accelerator for deep convolutional neural networks. In 2016 IEEE International Solid-State Circuits Conference (ISSCC); San Francisco, CA, USA, 31 Jan.-4 Feb. 2016; IEEE: 2016; pp. 262-263.

6. Moons, B.; Verhelst, M. An Energy-Efficient Precision-Scalable ConvNet Processor in $40 \mathrm{~nm}$ CMOS. IEEE Journal of Solid-State Circuits 2017, 52,903-914,doi:10.1109/JSSC. 2016.2636225. 